\title{
DESEMPENHO DE GENÓTIPOS DE NOVILHOS PARA ABATE AOS CATORZE MESES, GERADOS POR FÊMEAS DE DOIS ANOS ${ }^{1}$
}

\author{
JOÃO RESTLE ${ }^{2}$, IVAN LUIZ BRONDANI' ${ }^{3}$, JORGE LUIS CARVALHO FLORES ${ }^{3}$, \\ FABIANO NUNES VAZ ${ }^{4}$ e CLÁUDIO ANTUNES ${ }^{5}$
}

\begin{abstract}
RESUMO - O objetivo deste trabalho foi avaliar o desempenho de machos Hereford $(\mathrm{H})$, $1 / 2$ Jersey $(\mathrm{J}) 1 / 2 \mathrm{H}$ e $5 / 8 \mathrm{H} 3 / 8$ Nelore $(\mathrm{N})$, gerados por fêmeas acasaladas aos 14 meses de idade, confinados do desmame, aos sete meses, até o abate aos 14 meses, quando atingiram em média $195 \mathrm{~kg}$ de carcaça. A relação volumoso:concentrado foi decrescente, iniciando em 70:30 e finalizando em 40:60. O volumoso utilizado incluiu silagem de sorgo forrageiro, cana-de-açúcar e feno de aveia. Os consumos diários de matéria seca e de energia digestível dos três grupos de novilhos não diferiram significativamente, mas os novilhos $1 / 2 \mathrm{~J} 1 / 2 \mathrm{H}$ apresentaram maior consumo de matéria seca por $100 \mathrm{~kg}$ de peso vivo em relação aos outros dois grupos. Não houve diferença no ganho de peso médio diário durante os 193 dias de confinamento. A eficiência de conversão, expressa em Mcal de energia digestível e em quilogramas de matéria seca, para cada quilograma de ganho de peso, também não diferiu significativamente entre os três grupos testados.
\end{abstract}

Termos para indexação: Braford, Hereford, Jersey, Nelore, confinamento.

\section{PERFORMANCE OF GENOTYPES OF STEERS FOR SLAUGHTER AT FOURTEEN MONTHS, PRODUCED BY FEMALES OF TWO YEARS}

\begin{abstract}
The objective of the experiment was to study the feedlot performance of Hereford $(\mathrm{H})$, $1 / 2$ Jersey $(\mathrm{J}) 1 / 2 \mathrm{H}$ and $5 / 8 \mathrm{H} 3 / 8$ Nellore $(\mathrm{N})$ steers, produced by females bred at 14 months of age, confined from weaning to slaughter at 14 months of age when they reached average carcass weight of $195 \mathrm{~kg}$. The roughage:concentrate ratio was 70:30 at the beginning and declined to 40:60 at the end of the trial. The roughages used were sorghum silage, chopped sugar cane and oats hay. Daily dry matter and digestible energy intake of the three genetic groups were similar, but the $1 / 2 \mathrm{~J} 1 / 2 \mathrm{H}$ showed higher dry matter intake per $100 \mathrm{~kg}$ of live weight than the $\mathrm{H}$ and $5 / 8 \mathrm{H} 3 / 8 \mathrm{~N}$. No significant difference for average daily weight gain occurred during the 193 days feedlot period. Food efficiency, expressed in Mcal of digestible energy and kilograms of dry matter, per kilogram of weight gain also did not differ among the genetic groups.
\end{abstract}

Index terms: Braford, Hereford, Jersey, Nellore, feedlot.

\section{INTRODUÇÃO}

Em sistemas de exploração de pecuária de corte intensiva onde se busca cada vez mais a redução da idade de abate dos machos e de entoure das novilhas, a precocidade é de fundamental importância.

\footnotetext{
${ }^{1}$ Aceito para publicação em 3 de dezembro de 1998.

${ }^{2}$ Eng. Agr., Ph.D, Prof. Titular, Dep. de Zootecnia, UFSM CEP 97119-900 Santa Maria, RS. Bolsista do CNPq. E-mail: restle@infoway.com.br

${ }^{3}$ Zoot., M.Sc., UFSM.

${ }^{4}$ Zoot., Aluno do curso de Pós-Graduação, UFSM.

${ }^{5}$ Médico Veterinário, Dep. de Zootecnia, UFSM.
}

A raça Hereford pela sua precocidade e desenvolvimento vem sendo utilizada no Rio Grande do Sul nos sistemas de produção intensiva. Por meio da utilização de genótipos precoces e de pastagens cultivadas de inverno, consegue-se nas fêmeas o desenvolvimento necessário para que sejam acasaladas a partir dos 14 meses de idade, parindo pela primeira vez com idade média de dois anos. No entanto, mesmo com a utilização de pastagens cultivadas não se consegue nos machos o desenvolvimento e acabamento adequado para o abate aos 14 meses de idade. Neste caso, o confinamento surge como principal alternativa para suprir as exigências necessárias para que os machos atinjam condições 
de abate aos 14 meses (Restle \& Vaz, 1997; Restle et al., 1999).

O principal problema decorrente do acasalamento de fêmeas aos 14 meses é a ocorrência de problemas de distocia em função da reduzida área pélvica nestas fêmeas. Uma solução para reduzir este problema é a fecundação destas fêmeas utilizando touros de raças de pequeno porte, como o Jersey (Santiago, 1991). Os terneiros provenientes desse cruzamento não apresentam finalidade reprodutiva dentro da propriedade sendo destinados ao abate. Segundo Williams et al. (1995), que trabalharam com simulação em nove sistemas de produção diferentes, os animais cruza Jersey necessitariam de menor quantidade de energia metabolizável para ganhar um quilograma de peso de corpo vazio do que os animais Hereford definidos.

Outro genótipo empregado nos sistemas intensivos de exploração no Sul do país é o produto do cruzamento entre fêmeas Hereford com touros de raças zebuínas, atingindo-se na terceira geração de cruzamento alternado, os animais 5/8 Hereford 3/8 zebu, que além de um alto nível de heterozigose, aliam características desejáveis do Hereford e do zebuíno. No entanto, são poucos os trabalhos que compararam o desempenho desses animais com os definidos, e quase inexistentes são os trabalhos que compararam animais oriundos de sistemas de exploração intensiva, em que os terneiros gerados por fêmeas acasaladas aos 14 meses, destinam-se ao abate aos 14 meses.

Este trabalho teve como objetivo avaliar, em condições de exploração intensiva, com terminação aos 14 meses, as características de desempenho em confinamento dos machos resultantes do cruzamento entre Jersey e Hereford, Nelore e Hereford, assim como compará-los com animais contemporâneos da raça Hereford, sendo todos esses animais gerados por fêmeas acasaladas aos 14 meses de idade.

\section{MATERIAL E MÉTODOS}

O experimento foi conduzido de junho a dezembro de 1994, no Setor de Gado de Corte do Departamento de Zootecnia da Universidade Federal de Santa Maria, RS. Foram comparados bezerros de três grupos genéticos: Hereford (H), $1 / 2$ Jersey $1 / 2$ H e 5/8 H 3/8 Nelore, filhos de fêmeas acasaladas aos 14 meses, desmamados e castrados aos sete meses. Os bezerros, oito de cada grupo genético, foram tomados ao acaso do rebanho da Estância Guatambu, localizada no município de Dom Pedrito, RS, e submetidos sempre às mesmas condições de manejo e nutrição.

Os bezerros de cada grupo genético foram desverminados e distribuídos em dois lotes de quatro animais, e alojados em baias parcialmente cobertas. Os animais foram adaptados ao manejo e alimentação ao cocho durante 21 dias (período pré-experimental). Após esse período os terneiros foram pesados (peso inicial), dando início ao período experimental, que teve duração de 193 dias, dividido em seis períodos de 28 dias e um período de 25 dias. Os terneiros foram arraçoados duas vezes ao dia, sendo a metade da dieta fornecida pela manhã ( $8 \mathrm{~h})$ e o restante à tarde (16h). Diariamente, pela manhã, foram retiradas e pesadas as sobras de alimento do dia anterior, para ajuste da quantidade de alimento a ser oferecido e posterior cálculo de consumo

A dieta, comum aos três tratamentos, continha $12 \%$ de proteína bruta durante todo o período experimental. O volumoso utilizado incluiu silagem de sorgo forrageiro, cana-de-açúcar e feno de aveia. O concentrado incluiu milho em grão moído, farelo de soja, farelo de arroz desengordurado, farinha de carne, calcário calcítico, sal comum e bicarbonato de sódio. A relação volumoso:concentrado foi decrescente, iniciando em 70:30, 65:35, 60:40, 55:45, 45:55, 40:60 e 40:60, respectivamente, do 1 o ao 7o período. Na Tabela 1 é apresentada a formulação das dietas utilizadas nos diferentes períodos.

Antes das pesagens os novilhos eram submetidos a um jejum de sólidos de 14 horas. O controle de ectoparasitas foi efetuado conforme o grau de infestação dos animais e as vacinações seguiram o calendário utilizado pelo Departamento de Zootecnia da UFSM, conforme as recomendações da Secretaria de Agricultura do Rio Grande do Sul.

Durante o período experimental, a cada duas semanas foram coletadas amostras de todos os constituintes da dieta, para serem posteriormente analisadas, sendo determinada a matéria seca (MS), proteína bruta (PB) e a digestibilidade in vitro da MS e matéria orgânica. A energia digestível foi estimada pela fórmula indicada pelo Agricultural Research Council (1980).

O tipo de delineamento experimental utilizado foi o inteiramente casualizado, com duas repetições (lotes) por tratamento, utilizando-se o seguinte modelo matemático: $Y_{i j}=\mu+T_{i}+e_{i j}$, em que:

$Y_{i j}=$ observação $\mathrm{j}$ do animal que recebeu o tratamento i; $\mu=$ média geral; 
$T_{i}=$ efeito do tratamento $\mathrm{i}$;

$e_{i j}=$ erro aleatório associado a cada observação.

Os efeitos de período e da interação entre grupo genético e períodos também foram testados, e, por não serem significativos, foram removidos do modelo final. As médias foram submetidas à análise de variância e teste $\mathrm{F}$ a 5\%. As médias que apresentaram diferença significativa foram comparadas pelo teste PDIFF.

\section{RESULTADOS E DISCUSSÃO}

Na Tabela 2 é apresentado o resumo das análises de variância para as diferentes características estudadas. Na Tabela 3 encontram-se os resultados referentes a peso vivo inicial, peso vivo final, ganho de peso médio diário, conversão alimentar e consumo diário de matéria seca por animal, por percentagem de peso vivo e por unidade de tamanho metabólico. Os animais $1 / 2 \mathrm{~J} 1 / 2 \mathrm{H}$ apresentaram o menor peso inicial, o que é explicado pelo menor porte da raça Jersey, que contribuiu com a metade dos gens. Como não houve diferença entre os grupos genéticos no ganho de peso médio diário durante o período de confinamento, os animais $1 / 2 \mathrm{~J} 1 / 2 \mathrm{H}$ também apresentaram menor peso aos 14 meses.
Observa-se na Tabela 3 que o ganho de peso médio diário dos animais He 5/8 H 3/8 N não diferiu significativamente. Resultados similares são relatados por Flores (1997) em animais inteiros confinados do desmame ao abate aos 14 meses $(\mathrm{H}=1,326 \mathrm{~kg}$ e $5 / 8 \mathrm{H} 3 / 8 \mathrm{~N}=1,283 \mathrm{~kg}$ ). Crockett (1973) também obteve ganho de peso similar em confinamento, para novilhos $\mathrm{He}^{3 / 4} \mathrm{H}_{1}^{1} / 4$ Brahman (B), respectivamente, 1,053 e $1,135 \mathrm{~kg}$, quando previamente suplementados na recria, em pastagem, com uma quantidade equivalente a $0,5 \%$ do peso vivo. Quando o nível de suplementação na fase de recria aumentou para $1 \%$ do peso vivo, o mesmo autor verificou um maior ganho de peso no confinamento para os novilhos $\mathrm{H}$ $(1,253 \mathrm{~kg})$ do que para os $3 / 4 \mathrm{H} 1 / 4 \mathrm{~B}(1,112 \mathrm{~kg})$. Utilizando um nível de energia mais elevado ao empregado neste trabalho, para novilhos confinados dos 11 aos 15 meses, Sherbeck et al. (1995) observaram que o ganho de peso decresceu à medida que aumentou o percentual de sangue zebuíno e decresceu a porcentagem de sangue $\mathrm{H}$. Segundo Moore et al. (1975) com melhores níveis alimentares, regulados pelo conteúdo energético da dieta $(3,075 \mathrm{Mcal} / \mathrm{kg}$ MS consumida), animais $\mathrm{H}$ apresentaram melhor digestibilidade da MS consumida e maior retenção

TABELA 1. Composição percentual da matéria seca da dieta oferecida nos diferentes períodos experimentais.

\begin{tabular}{|c|c|c|c|c|c|c|c|c|}
\hline \multirow[t]{2}{*}{ Alimento } & \multicolumn{7}{|c|}{ Período } & \multirow[t]{2}{*}{ Média } \\
\hline & 1 & 2 & 3 & 4 & 5 & 6 & 7 & \\
\hline Silagem de sorgo forrageiro ${ }^{1}$ & 70,00 & 65,00 & 50,40 & 36,85 & 31,15 & 31,54 & 33,89 & 45,55 \\
\hline Cana-de-açúcar & 0,00 & 0,00 & 9,60 & 18,15 & 6,44 & 0,00 & 0,00 & 4,88 \\
\hline Feno de aveia & 0,00 & 0,00 & 0,00 & 0,00 & 7,41 & 13,46 & 6,11 & 3,85 \\
\hline Farelo de arroz desengordurado & 8,37 & 11,45 & 12,98 & 21,86 & 15,14 & 0,00 & 0,00 & 9,97 \\
\hline Farelo de soja & 16,14 & 0,00 & 0,00 & 0,00 & 8,82 & 10,34 & 10,86 & 6,59 \\
\hline Farinha de carne & 0,00 & 17,08 & 19,56 & 18,95 & 8,82 & 6,38 & 6,72 & 11,07 \\
\hline Grão de milho moído & 3,15 & 5,63 & 6,40 & 3,15 & 17,64 & 36,14 & 37,92 & 15,72 \\
\hline Calcário calcítico & 2,04 & 0,00 & 0,00 & 0,00 & 2,03 & 1,22 & 1,28 & 0,94 \\
\hline Cloreto de sódio & 0,30 & 0,35 & 0,53 & 0,54 & 0,58 & 0,49 & 0,51 & 0,47 \\
\hline Bicarbonato de sódio & 0,00 & 0,49 & 0,53 & 0,54 & 0,57 & 0,46 & 0,00 & 0,37 \\
\hline Composto vitamínico/antibiótico ${ }^{2}$ & 0,00 & 0,00 & 0,00 & 0,00 & 1,37 & 0,00 & 2,70 & 0,58 \\
\hline Energia digestível, Mcal/kg MS & 2,504 & 2,283 & 2,332 & 2,340 & 2,576 & 2,880 & 2,899 & 2,545 \\
\hline
\end{tabular}

${ }^{1}$ Agroceres (AG 2002).

${ }^{2}$ Rumensin. 
TABELA2. Quadrados médios (QM) do modelo e residual, teste F e coeficiente de determinação para as características de desempenho de novilhos de diferentes grupos genéticos, confinados do desmame ao abate aos 14 meses.

\begin{tabular}{|c|c|c|c|c|c|c|}
\hline \multirow[t]{2}{*}{ Característica } & \multicolumn{2}{|c|}{ Graus de liberdade } & \multicolumn{2}{|c|}{ QM } & \multirow[t]{2}{*}{$\mathrm{F}$} & \multirow[t]{2}{*}{$\mathrm{R}^{2}$} \\
\hline & Modelo & Erro & Modelo & Erro & & \\
\hline Peso inicial & 2 & 3 & 188,2 & 1,0 & 188,17 & 0,99 \\
\hline Peso final & 2 & 3 & 528,7 & 160,7 & 3,29 & 0,69 \\
\hline Ganho de peso médio diário & 2 & 3 & 0,003 & 0,005 & 0,60 & 0,28 \\
\hline Consumo de MS & 2 & 3 & 0,100 & 0,046 & 2,16 & 0,59 \\
\hline Cons. MS/100 kg PV & 2 & 3 & 0,078 & 0,007 & 10,89 & 0,88 \\
\hline Cons. MS/PV ${ }^{0,75}$ & 2 & 3 & 0,088 & 0,010 & 8,86 & 0,86 \\
\hline Conversão alimentar & 2 & 3 & 0,255 & 0,187 & 1,37 & 0,48 \\
\hline Cons. energia digestível (ED) & 2 & 3 & 0,212 & 0,723 & 0,29 & 0,16 \\
\hline Cons. ED/PV & 2 & 3 & 0,112 & 0,064 & 1,76 & 0,54 \\
\hline Cons. ED/kg ganho PV & 2 & 3 & 0,344 & 0,664 & 0,52 & 0,26 \\
\hline
\end{tabular}

TABELA 3. Média para peso vivo inicial e final, ganho de peso médio diário, consumo diário de matéria seca por animal, por $100 \mathrm{~kg}$ de peso vivo (PV) e por unidade de tamanho metabólico ( $\left.\mathrm{PV}^{0,75}\right)$, e conversão alimentar de novilhos de diferentes grupos genéticos, confinados do desmame ao abate aos 14 meses.

\begin{tabular}{lccccc}
\hline \multirow{2}{*}{ Característica } & \multicolumn{3}{c}{ Grupo genético } & \multirow{2}{*}{$\mathrm{CV}^{1}$} & \multirow{2}{*}{$\mathrm{P}>\mathrm{F}$} \\
\cline { 2 - 4 } & $\mathrm{H}$ & $1 / 2 \mathrm{~J} \mathrm{1} / 2 \mathrm{H}$ & $5 / 8 \mathrm{H} \mathrm{3/8} \mathrm{N}$ & $(\%)$ & \\
\hline Peso inicial, kg & 170 & 152 & 168 & 9,37 & 0,0569 \\
Peso final, kg & 380 & 347 & 371 & 7,96 & 0,0847 \\
Ganho de peso médio diário, kg & 1,09 & 1,01 & 1,05 & 6,97 & 0,6057 \\
Consumo/animal, kg MS & 7,37 & 7,42 & 7,01 & 2,97 & 0,2620 \\
Consumo/100 kg PV, kg MS & $2,68 \mathrm{a}$ & $2,97 \mathrm{~b}$ & $2,60 \mathrm{a}$ & 3,08 & 0,0421 \\
Consumo/PV & 109 & 118 & 105 & 2,84 & 0,0551 \\
Conv. alimentar, kg MS & 6,78 & 7,36 & 6,71 & 6,21 & 0,3783 \\
\hline
\end{tabular}

${ }^{1}$ Coeficiente de variação.

2 Médias seguidas por letras distintas diferem entre si a $5 \%$ de probabilidade, pelo teste PDIFF.

de $\mathrm{N}$ do que animais Brahman, Aberdeen Angus, Shorthorn e Santa Gertrudis. No entanto, sob um nível baixo de energia (2,275 Mcal/kg MS consumida) os animais $\mathrm{H}$ apresentaram menores valores em relação a essas características que as demais raças estudadas. É provável que a similaridade no ganho de peso entre os animais $\mathrm{H}$ e $5 / 8 \mathrm{H} 3 / 8 \mathrm{~N}$ se deve, em parte, ao nível energético $(2,545 \mathrm{Mcal} / \mathrm{kg} \mathrm{MS}$ oferecida) da dieta utilizada no presente experimento, e que foi intermediário aos dois níveis empregados por Moore et al. (1975).
$\mathrm{Na}$ comparação entre os animais $1 / 2 \mathrm{~J} 1 / 2 \mathrm{H}$ e $\mathrm{H}$, observa-se uma diferença não significativa de $7,9 \%$ no ganho de peso médio diário, a favor dos últimos. Considerando que as raças leiteiras não sofreram seleção para aumento da velocidade de ganho de peso, poderia-se esperar uma maior diferença no ganho de peso médio diário a favor dos animais $\mathrm{H}$, por se tratar de uma raça de corte com intensa seleção em velocidade de crescimento. No entanto, Hentges et al. (1973), ao traçarem considerações sobre o desempenho de novilhos de raças de corte 
definidas em relação a novilhos provenientes do cruzamento destas com raças leiteiras, citam que não foi verificado menor velocidade de ganho de peso nos animais cruzados, no entanto deve ser considerado que esse autor trabalhou com uma raça leiteira de maior porte (Holandês).

Wilham (1973), ao trabalhar com animais cruza Jersey, relatou que, para a terminação de animais com idade média de um ano, esse cruzamento tornou-se interessante pela qualidade do produto e pela presença de um adequado grau de acabamento para os padrões americanos. Nesse mesmo trabalho, comenta-se que os animais cruza raça leiteira pecam pela pior conversão alimentar que apresentam, o que no presente trabalho não foi verificado, embora tenha ocorrido uma diferença de $8,55 \%$ entre os novilhos cruza J e os H definidos. Diferença similar na conversão alimentar $(7,65 \%)$ foi verificada por Hargrove et al. (1983), ao trabalhar com animais Angus $(5,62)$ comparados com animais $1 / 2$ Aberdeen Angus 1/2 Pardo Suíço $(6,05)$.

$\mathrm{Na}$ comparação entre $5 / 8 \mathrm{H} 3 / 8 \mathrm{~N}$ e $\mathrm{H}$, também não verificou-se diferença $(\mathrm{P}>0,05)$ na conversão alimentar, sendo de 6,71 e 6,78, na mesma ordem. Flores (1997) verificou conversão alimentar de 5,63 e 5,40, respectivamente, para 5/8 H 3/8 N e H. Restle \& Vaz (1997) relataram que quando ocorre maior velocidade de ganho de peso dos animais cruzados em relação aos definidos, devido à heterozigose, essa diferença é resultado de um maior consumo de alimentos dos cruzados e não de uma melhor eficiência alimentar.

Na comparação entre o consumo de alimentos dos novilhos dos três grupos genéticos, verificou-se que os bezerros $1 / 2 \mathrm{~J} 1 / 2 \mathrm{H}$ apresentaram um maior consu- mo $(\mathrm{P}<0,0421)$ em relação aos $\mathrm{H}$ e $5 / 8 \mathrm{H} 3 / 8 \mathrm{~N}$, quando esse foi expresso em $100 \mathrm{~kg}$ de peso vivo $(\mathrm{H}=2,68 \mathrm{~kg} ; 1 / 2 \mathrm{~J} 1 / 2 \mathrm{H}=2,97 \mathrm{~kg}$; e $5 / 8 \mathrm{H} 3 / 8 \mathrm{~N}=2,60 \mathrm{~kg}$ ). Ao expressar o consumo para unidade de tamanho metabólico, o consumo foi de 109, 118 e $105 \mathrm{~g}$, respectivamente, para $\mathrm{H}, 1 / 2 \mathrm{~J} 1 / 2 \mathrm{He} 5 / 8 \mathrm{H} 3 / 8 \mathrm{~N}(\mathrm{P}>0,0551)$.

Valores inferiores aos do presente experimento, no consumo de MS, foram relatados por Flores (1997) em animais confinados dos sete aos 14 meses. Esse autor verificou um consumo de MS, expresso em $100 \mathrm{~kg}$ de peso vivo de $2,32 \mathrm{~kg} /$ dia para animais $\mathrm{H}$ e 2,22 kg para animais $5 / 8 \mathrm{H} 3 / 8 \mathrm{~N}$, o que também foi observado quando o consumo foi expresso para peso metabólico, sendo de 97,1 e 94,4, respectivamente.

O maior consumo de MS verificado nos animais $1 / 2 \mathrm{~J} 1 / 2 \mathrm{H}$ foi uma conseqüência da seleção para consumo de alimentos que as raças leiteiras, entre elas a Jersey, sofreram durante a sua formação. Hentges et al. (1973) e Hargrove et al. (1983) avaliando novilhos produtos do cruzamento entre raças de corte britânicas e leiteiras de grande porte, também verificaram maior consumo de MS em relação aos animais das raças de corte definidos.

Na Tabela 4, também são apresentados os resultados referentes a consumo de energia digestível, expresso em Mcal/animal/dia, kcal/PV ${ }^{0,75}$ e em $\mathrm{Mcal} / \mathrm{kg}$ de ganho de peso vivo. Essas formas de expressão do consumo de alimentos e conversão tornam-se mais precisas ao considerar-se que a relação de energia da dieta variou nos diferentes períodos. Verifica-se nessa tabela que o consumo de energia por animal não apresentou diferença $(\mathrm{P}>0,7654)$. Resultado semelhante é citado por Flores (1997) na comparação entre H e 5/8 H 3/8 N (22,7 e 22,8 Mcal, respectivamente). No presente trabalho também não

TABELA 4. Médias do consumo diário de energia digestível por animal, por unidade de tamanho metabólico e por quilograma de ganho de peso vivo de novilhos de diferentes grupos genéticos, confinados do desmame ao abate aos 14 meses.

\begin{tabular}{lccccc}
\hline \multirow{2}{*}{ Consumo de } & \multicolumn{3}{c}{ Grupo genético } & \multirow{2}{*}{$\mathrm{CV}^{1}$} & \multirow{2}{*}{$\mathrm{P}>\mathrm{F}$} \\
\cline { 2 - 4 } & $\mathrm{H}$ & $1 / 2 \mathrm{~J} 1 / 2 \mathrm{H}$ & $5 / 8 \mathrm{H} \mathrm{3/8} \mathrm{N}$ & $(\%)$ & \\
\hline Energia digestível/cabeça, Mcal & 20,20 & 19,55 & 19,85 & 4,28 & 0,7654 \\
Energia digestível/PV ${ }^{0,75}, \mathrm{kcal}$ & 299 & 311 & 298 & 22,64 & 0,3126 \\
En. digestível/kg ganho PV, Mcal & 18,58 & 19,41 & 18,95 & 4,29 & 0,6404 \\
\hline
\end{tabular}


se verificou diferença no consumo de energia digestível ajustado para peso metabólico, sendo de 299,311 e $298 \mathrm{kcal}$, respectivamente, para $\mathrm{H}, 1 / 2 \mathrm{~J} 1 / 2 \mathrm{H}$ e $5 / 8 \mathrm{H} 3 / 8 \mathrm{~N}(\mathrm{P}>0,3126)$, valores bastante similares aos encontrados por Flores (1997), de 308 e $299 \mathrm{kcal}$ para $\mathrm{He} 5 / 8 \mathrm{H} 3 / 8 \mathrm{~N}$.

O consumo de energia digestível para cada quilo de ganho de peso foi similar entre os tratamentos (P>0,6404). Flores verificou 17,1 e 17,8 Mcal/kg ganho de peso, respectivamente, para $\mathrm{H}$ e $5 / 8 \mathrm{H}$ $3 / 8 \mathrm{~N}$. No presente estudo, a leve diferença que os animais $1 / 2 \mathrm{~J} 1 / 2 \mathrm{H}$ apresentaram em relação aos $\mathrm{H}$, provavelmente foi devido à diferença da composição do ganho de peso, onde os animais $1 / 2 \mathrm{~J} 1 / 2 \mathrm{H}$ apresentaram maior porcentagem de gordura na carcaça em relação aos definidos $(20,05$ vs $18,45 \%)$.

\section{CONCLUSÕES}

1. O consumo de alimentos expresso em $100 \mathrm{~kg}$ de peso vivo é maior em animais $1 / 2$ Jersey $1 / 2$ Hereford, em comparação aos Hereford e $5 / 8$ Hereford $3 / 8$ Nelore.

2. Não há diferença no ganho de peso, conversão alimentar e consumo de energia entre Hereford, $1 / 2$ Jersey $1 / 2$ Hereford e 5/8 Hereford 3/8 Nelore.

\section{AGRADECIMENTOS}

Ao Prof. José Henrique Souza da Silva, pelo auxílio nas análises estatísticas.

\section{REFERÊNCIAS}

AGRICULTURAL RESEARCH COUNCIL. The nutrient requirements of ruminant livestock. Surrey: The Gresham Press, 1980.351p.

CROCKETT, J.R. Terminacion de terneros en un sistema de cruzamiento alternado. In: KOGER, M.; CUNHA, T.J.; WARNICK, A.C. (Eds.). Cruzamientos en ganado vacuno de carne. Montevideo: Hemisferio Sur, 1973. p.351-363.

FLORES, J.L.C. Desempenho em confinamento e características de carcaça e da carne de machos inteiros de diferentes grupos genéticos na fase do desmame ao abate aos catorze meses. Santa
Maria, RS: Universidade Federal de Santa Maria, 1997. 109p. Tese de Mestrado.

HARGROVE, D.D.; RESTLE, J.; EUCLIDES, K.; OLSON, T.A.; KOGER, M. Feedlot performance and carcass characteristics of Angus, Brown Swiss, F1, F2, and backcross steers. Florida Beef Cattle Research Report, Gainesville, v.1, n.1, p.18-24, 1983

HENTGES, J.F.; FRANKE, D.E.; PALMER, A.Z. Novillos de razas de carne $\mathrm{x}$ lecheras, del destete a la faena. In: KOGER, M.; CUNHA, T.J.; WARNICK, A.C. (Eds.). Cruzamientos en ganado vacuno de carne. Montevideo: Hemisferio Sur, 1973. p.389407.

MOORE, R.L.; ESSING, H.W.; SMITHSON, L.J. Influence of breeds of beef cattle on ration utilization. Journal of Animal Science, Champaign, v.41, n.1, p.203-207, Jan. 1975

RESTLE, J.; VAZ, F.N. Aspectos quantitativos da carcaça de machos Hereford, inteiros ou castrados, abatidos aos catorze meses. Pesquisa Agropecuária Brasileira, Brasília, v.32, n.10, p.1091-1095, out. 1997.

RESTLE, J.; VAZ, F.N.; BRONDANI, I.L.; GONÇALVES, J.M.; ANDREATTA, E. Estudo da carcaça de machos Braford desmamados aos 72 ou 210 dias, abatidos aos catorze meses. Pesquisa Agropecuária Brasileira, Brasília, v.34, n.11, p.2137-2144, nov. 1999.

SANTIAGO, A.A. Os cruzamentos na pecuária bovina. Campinas: Instituto Campineiro de Ensino Agrícola, 1991. 549p.

SHERBECK, J.A.; TATUM, T.G.; FIELD, J.B.; MORGAN, J.B.; SMITH, G.C. Feedlot performance, carcass traits, and palatability traits of Hereford and Hereford x Brahman steers. Journal of Animal Science, Champaign, v.73, n.12, p.3613-3620, Dec. 1995.

WILHAM, R.L. Effectos del cruzamiento sobre la eficiencia del engorde a corral: razas de carne $\mathrm{x}$ razas lecheras. In: KOGER, M.; CUNHA, T.J.; WARNICK, A.C. (Eds.). Cruzamientos en ganado vacuno de carne. Montevideo: Hemisferio Sur, 1973. p.383-388.

WILLIAMS, C.B.; BENNETT, G.L.; KEELE, J.W. Simulated influence of postweaning production system on performance of different biological types of cattle. III. Biological efficiency. Journal of Animal Science, Champaign, v.73, n.2, p.686-698, Feb. 1995 